\title{
MUJERES EN LAS SERIES POLÍTICAS CONTEMPORÁNEAS. ¿UNA GEOGRAFÍA COMÚN DE SU PRESENCIA EN LA ESFERA PÚBLICA?
}

\author{
Women in contemporary political series: A shared \\ geography of their presence in the public sphere?
}

Anna Tous-Rovirosa y Sue Aran-Ramspott

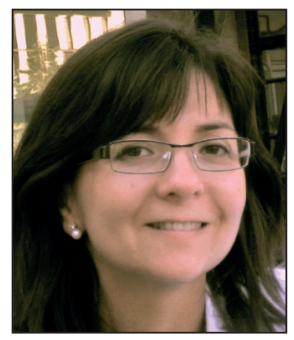

Anna Tous-Rovirosa es profesora agregada del Departamento de Periodismo y Ciencias de la Comunicación de la Universitat Autònoma de Barcelona (UAB). Editora del libro La política en las series de televisión (UOC, 2015). Ganadora del II Premio del Consell Audiovisual de Catalunya (2008). Ha sido profesora visitante de las universidades Rühr (Bochum, Alemania) y Federal de Bahía (Brasil). Directora de la Revista Anàlisi, sus principales líneas de investigación son la ficción televisiva, la narrativa audiovisual, la convergencia mediática, y el periodismo en internet. Es miembro de los grupos de investigación reconocidos Ofent y Localcom, de la UAB. http://orcid.org/0000-0003-4519-3793

Universitat Autònoma de Barcelona, Facultad de Ciencias de la Comunicación Campus UAB. C/ de la Vinya, s/n. 08193 Bellaterra (Barcelona), España anna.tous@uab.cat

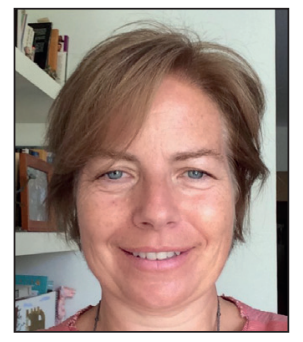

Sue Aran-Ramspott es profesora titular de Comunicación Audiovisual en la Universitat Ramon Llull. Miembro del grupo de investigación reconocido Digilab, Strategy, Media and Regulation (URL). Primer premio de investigación en comunicación (CAC, Consejo del Audiovisual de Catalunya, 2008). Miembro del Consejo Asesor de la Corporación Catalana de Medios Audiovisuales. Profesora visitante en la University of London. Sus principales líneas de investigación son teorías de la recepción, jóvenes espectadores, representación de la violencia y de las relaciones amorosas, ficción audiovisual, series, YouTube e identidades.

http://orcid.org/0000-0001-6664-0172

Universitat Ramon Llull Facultad de Comunicación y Relaciones Internacionales Blanquerna Plaza Joan Corominas, s/n. 08001 Barcelona, España suear@blanquerna.url.edu

\section{Resumen}

La actual ficción seriada presenta un elevado número de producciones dedicadas a la política en el mundo occidental. La mayoría tienen como características comunes una representación fictiva de la política de su propio entorno. A partir del modelo de Hallin y Mancini $(2004,2012)$, esta investigación se interroga sobre la vigencia de un "mapa" temático-geográfico de las series políticas, correlacionado con las representaciones de los personajes femeninos. El análisis temático de seis series nos permite observar si la ficción seriada de temática política producida a nivel internacional pretende dar mayor protagonismo a los personajes femeninos de acuerdo con la voluntad de superación de los estereotipos de género. Los resultados señalan la pervivencia de personajes femeninos que se adscriben a una esfera más íntima que pública, aunque se identifican diferencias notables en relación con el origen geográfico de las producciones.

\section{Palabras clave}

TV; Televisión; Series; Ficción; Política; Género; Mujer; Comunicación y género; Personajes femeninos; Estereotipos.

\section{Abstract}

There are currently a large number of television series which focus on politics in the Western world. Most of these shows present a fictionalized account of politics in the local region or country. Using the Hallin and Mancini model (2004, 2012) as a starting point, this paper discusses the validity of a thematic geographic map for political shows, and correlates the map with the way female characters are represented. A thematic analysis of six series allows the researchers to observe whether or not internationally-produced series on political themes try to give greater importance to female characters in an attempt to overcome gender stereotyping. The results of this observation point to the continued existence of female characters 
appearing more in the private sphere than the public one. There are, however, differences between the television series that can be connected to the geographic location where they were produced.

\section{Keywords}

TV; Television; Series; Fiction; Politics; Gender; Women; Communication and gender; Female characters; Stereotypes.

Tous-Rovirosa, Anna; Aran-Ramspott, Sue (2017). “Mujeres en las series políticas contemporáneas. ¿Una geografía común de su presencia en la esfera pública?”. El profesional de la información, v. 26, n. 4, pp. 684-694.

https://doi.org/10.3145/epi.2017.jul.12

\section{Introducción}

En el origen de este artículo subyace una pregunta: ¿qué nos dice la ficción sobre la política? Más concretamente, ¿̇e pueden analizar las series de televisión de tema político en clave geopolítica? Entendiendo la geopolítica como la disciplina que aborda la situación política de un territorio y la asocia con la geografía en que se encuentra, nuestro interés es observar desde las ciencias de la comunicación cómo la serialidad televisiva con tramas políticas interacciona, subraya o renueva los modelos políticos en los que geográficamente se enmarcan las series.

\section{Las series políticas con protagonismo de mujer política son minoritarias, aunque tienen mayor presencia en los sistemas liberal y anglosajón}

Como objeto de estudio centramos nuestro análisis en la presencia de los personajes femeninos en dichas tramas, con la voluntad de reunir el debate sobre la representación de la mujer en un escenario global supuestamente atento a las políticas de género y con voluntad de superación de determinados estereotipos discriminatorios de los imaginarios sociales, tanto en la esfera mediática como en la pública.

Dentro de la eclosión de series políticas en la ficción televisiva reciente, podemos destacar los casos de algunas series centradas en la figura de la mujer. Las series analizadas tienen el precedente de Señora presidenta (2005-2006) y terminan en la contemporánea The good fight (2017).

\section{Marco teórico}

\subsection{Representación de la mujer en la ficción televisi- va seriada}

Mucho se ha hablado de la influencia de los media como conformadores de un imaginario público, colectivo o social (Ang, 1985; Morley, 1986; Llull, 1990; Silverstone, 1994; Thompson, 1998).

Sangrador (1996) señala el enorme poder de influencia de los media a través de la transmisión de estereotipos o "guiones" interiorizados, sean seriales o series de ficción, películas, canciones...

Engelstad (2008, p. 309) señala, desde una aproximación crítica al conocimiento cultural, que ver drama en televisión, cine o teatro suele implicar el reconocimiento de los hechos tal y como se muestran, así como la emisión de cierto tipo de juicios (estéticos, morales, o de otro tipo) sobre la acción que los personajes llevan a cabo.

En ocasiones anteriores (Tous-Rovirosa; Meso-Ayerdi; Simelio-Solà, 2013, p. 71) hemos señalado que la ficción es uno de los géneros comunicativos que ofrece mayores posibilidades de mostrar la transformación social. La ficción favorece la conexión de los espectadores con tramas de grados diversos de anclaje en la realidad a partir de personajes y situaciones; es decir, desde la implicación cognitiva y sobre todo emocional de la audiencia con esas experiencias individuales o colectivas, que actúan también como transmisoras de valores. La denominada participación activa del público forma parte de la competición por el apego de los consumidores por los productos, como señalan Lacalle y Castro-Mariño (2016, p. 247), en términos de la actual economía de servicios. De manera notoria, la ficción televisiva seriada puede mostrar en profundidad un retrato de la esfera privada y pública a pesar de la imperiosa economía narrativa y la complejidad del entorno político y social.

Sin embargo, esa profundidad parece sesgada entre otros factores por el peso de los estereotipos de género. Tanto los discursos sobre política de género como la presencia y representación de mujeres y hombres en los medios reflejan muchos rasgos que recalcan las dificultades de la erradicación de la discriminación de género.

En general los personajes masculinos son mostrados como cultos, independientes y poderosos, y los femeninos como emocionales, dependientes, jóvenes, sexys y dominados por hombres

La investigación sobre género y comunicación en las series de ficción tiene una amplia tradición anglosajona (Gerbner et al., 1978; Durkin, 1985; Davis, 1990; Geraghty, 1991; Fouts; Burggraf, 1999), y española (Galán-Fajardo, 2007; Gordillo-Álvarez; Guarinos, 2010; Medina-Bravo et al., 2010; Aran-Ramspott; Medina-Bravo; Rodrigo-Alsina, 2011; García-Muñoz; Fedele; Gómez-Díaz, 2012).

Como observan Fernández-Villanueva et al. (2009), estudios en una diversidad de países muestran que los retratos e imágenes de los hombres tienden a ser más complejos que los de las mujeres. Según dicha investigación, enmarcada en el análisis de medios en distintos contextos culturales de 
EUA y Europa, incluyendo España, en general los personajes masculinos tienden a ser mostrados como cultos (o informados), independientes y poderosos, y los personajes femeninos como emocionales, dependientes, jóvenes, sexys y dominados por hombres.

Algunos años más tarde, esta polarización sigue presente. Tous-Rovirosa, Meso-Ayerdi y Simelio-Solà (2013, p. 71) señalan en estudios sobre la televisión esa perpetuación de clichés y estereotipos de género, donde perviven todavía entre otros aspectos la representación del hombre como dominante y la mujer como complementaria, menos inteligente y dedicada al cuidado de los demás, así como la tendencia a relegar los personajes femeninos a la esfera privada, dejando el ámbito del trabajo como un bastión básicamente masculino. Y como analizamos recientemente (Aran-Ramspott et al., 2014, p. 78), esta polarización estereotipada se reconoce en las series de ficción emitidas en España, tanto de producción americana como española.

Un repaso a la presencia de la mujer en la ficción televisiva estadounidense nos muestra algunas características relevantes a recordar: la incorporación de la mujer al mundo laboral, "televisivamente" hablando, ha sido paulatina y con una aceptación natural, desempeñando en la ficción papeles tradicionalmente atribuidos a hombres, ya sea por la dureza y la violencia verbal (The closer, TNT: 2005-2012) o las tácticas "mafiosas" (Daños y perjuicios, Damages, FX Network: 2007-2012) (Tous-Rovirosa, 2011). Asimismo, se observa una tendencia creciente a una representación cosificada de la mujer, convertida en IT-girl cuyo principal anhelo es gustar al sexo contrario, especialmente en series para público adolescente (Cougar town, ABC: 2009; Gossip girl, CBS: 2007-2012; Hellcats, CBS: 2010-11).

Una tendencia es la representación cosificada de la mujer, convertida en IT-girl cuyo principal anhelo es gustar al sexo contrario, especialmente en series para público adolescente

En una panorámica compleja como es la ficción televisiva contemporánea, que no tiene un modelo único, se observa una radicalización de las tendencias, bien sea la más cercana al feminismo (Girls, HBO: 2012-17; United States of Tara, Showtime: 2009-11; Transparent, Amazon Studios: 2014-; A dos metros bajo tierra, HBO: 2001-05) o las ya mencionadas de cosificación y culto al cuerpo.

La progresiva aceptación y normalización de las mujeres en el entorno laboral, ya sean médicas, detectives, forenses, abogadas o políticas es bastante generalizada en la ficción seriada occidental, pero presenta diferencias considerables según los países. Tal y como Lacalle y Gómez (2016) han señalado en relación con la ficción española, los cargos de responsabilidad femeninos se asocian frecuentemente con una caracterización negativa del personaje.

\subsection{Series políticas, modelos políticos}

A continuación se describe la tipología propuesta, en función de la adscripción geográfica en relación con las premisas de Hallin y Mancini (2004; 2012). De acuerdo con dichos autores, los modelos políticos de los países occidentales responden a tres sistemas:

$$
\begin{aligned}
& \text { - modelo mediterráneo } \\
& \text { - modelo liberal } \\
& \text { - modelo democrático-corporativo }
\end{aligned}
$$

Aunque las producciones audiovisuales que estamos analizando son de ficción y no documentales, suponen una representación de los poderes públicos y del sistema político del país productor de la serie y por extensión, del mencionado ámbito geográfico. Los productos televisivos a analizar aquí son productos fictivos (Searle, 1975) que reflejan la realidad política y social, muchas veces con la ayuda de asesores ${ }^{1}$ que complementan la tarea de los guionistas.

\section{A pesar de un mayor protagonismo de los personajes femeninos, persiste en las se- ries políticas una construcción de valores que adscribe los personajes femeninos a una esfera más íntima que pública}

Parafraseando a Gerbner et al. (1978, p. 18), cada sistema televisivo refleja el contexto histórico, político, social, económico y cultural en el que se desarrolla.

Según las premisas de Hallin y Mancini (2004; 2012):

- las series del modelo mediterráneo o polarizado se centran en la corrupción política (democratización tardía, clientelismo, un desarrollo más débil de la autoridad legal);

- las series del modelo democrático-corporativo (Europa central) muestran particularidades propias (un estado del bienestar más fuerte)

- las series del modelo liberal (nórdicas y anglosajonas) tienen un mayor abanico de temas y una prolongada tradición satírica (comedia, autocrítica).

Los países mediterráneos, polarizados, o bien proyectan una imagen positiva de la política en los dramas y comedias históricas o se sirven del drama como reflejo de la corrupción de estados de tradición democrática reciente, propia del clientelismo de los países mediterráneos (Tous-Rovirosa, 2017).

La tabla 1 muestra un resumen de las principales series políticas en relación con los sistemas y países. 
Tabla 1. Principales series políticas en relación con los sistemas y los países de producción

\begin{tabular}{|c|c|}
\hline Serie & Año \\
\hline \multicolumn{2}{|c|}{ Sistema liberal. Series estadounidenses } \\
\hline El Ala Oeste de la Casa Blanca & $N B C, 1999-2006$ \\
\hline 24 & FOX, 2001-2010 \\
\hline The wire & $H B O, 2002-2008$ \\
\hline Señora presidenta & $A B C, 2005-2006$ \\
\hline Parks and recreation & NBC, 2009-2015 \\
\hline The good wife & CBS, 2009-2016 \\
\hline Homeland & Showtime, 2011- \\
\hline Los Kennedy & Reelz Channel, 2011 \\
\hline Political animals & USA, 2012 \\
\hline Scandal & $A B C, 2012-$ \\
\hline Veep & $H B O, 2012-$ \\
\hline Alpha house & Amazon, 2013-2014 \\
\hline House of cards & Netflix, 2013- \\
\hline Madam secretary & CBS, 2014- \\
\hline The good fight & CBS, 2017- \\
\hline \multicolumn{2}{|c|}{ Sistema liberal. Series británicas } \\
\hline Vote, vote vote for Nigel Barton & $B B C, 1965$ \\
\hline Yes, minister & $B B C, 1980-1984$ \\
\hline Yes, primer minister & $B B C, 1986-1987$ \\
\hline The thick of it & $B B C, 2005-2012$ \\
\hline The red riding trilogy $(1974,1980,1983)$ & Channel 4, 2009 \\
\hline The gour & $B B C, 2011-2012$ \\
\hline Byw celwydd & S4C, 2016- \\
\hline \multicolumn{2}{|c|}{ Sistema democrático-corporativo. Noruega, Alemania } \\
\hline Borgen & Danmarks radio, 2010-2013 \\
\hline Occupied (Okkupert) & TV2, 2016- \\
\hline Mitten in Deutschland & NSU, 2016- \\
\hline \multicolumn{2}{|c|}{ Sistema mediterráneo. Italia, Francia, España } \\
\hline Gomorra & Sky Italia, 2014- \\
\hline $1992^{*}$ & La7, 2015 \\
\hline Marseille & Netflix, 2016 \\
\hline Baron noir & Canal plus, 2016- \\
\hline Señor alcalde & Tele 5, 1998 \\
\hline Moncloa, ¿dígame? & Tele 5, 2001 \\
\hline Cuéntame & TVE1, 2001- \\
\hline 23F El día más difícil del Rey & TVE1, 2009 \\
\hline Adolfo Suárez, el presidente & Antena 3, 2010 \\
\hline 14 de abril. La república & TVE1, 2011 \\
\hline Crematorio & Canal Plus, 2011 \\
\hline Isabel & TVE1, 2012-2014 \\
\hline Mario Conde, los días de gloria & Tele5, 2013 \\
\hline Carlos rey emperador & TVE1, 2015-2016 \\
\hline La embajada & Antena 3, 2016- \\
\hline
\end{tabular}

* 1992 tiene las secuelas 1993 (Sky, 2017) y 1994

\section{Representación de la mujer en las series políticas}

La política como subgénero dramático en las series de televisión ha experimentado un auge exponencial en la última década (López-García, 2015; Tous-Rovirosa, 2015; Van-Zoonen; Wring, 2012). El ascenso de la representación de la mujer en la política "real" ha ido acompañada de numerosos estudios en los últimos años, como los publicados por Richardson, Parry y Corner (2013); Campus (2013) y Horn-Sheeler y Vasby-Anderson (2013).

Estas investigaciones comparten la importancia del framing en referencia a la cobertura de determinadas minorías, como es el caso de la mujer en la política. Se considera que los avances de la presencia de la mujer en la política no tienen su correlato en su cobertura mediática. La perspectiva androcéntrica y patriarcal se mantiene hasta tal punto en la representación mediática que el rol de las mujeres políticas se convierte en una mediación continua entre su género y su deseado rol como líderes en la sociedad.

La progresiva normalización de las mujeres en el entorno laboral (médicas, detectives, forenses, abogadas o políticas), es bastante generalizada en la ficción seriada occidental, con diferencias por países

Los cambios políticos y sociales acontecidos en los últimos años incorporan como hecho relevante el liderazgo femenino en países en los que una mujer ha llegado a ocupar el máximo cargo ejecutivo (Brasil, Alemania, Reino Unido, Dinamarca, Islandia, Argentina). Pero la representación mediática de dichas líderes sigue siendo estereotipada (Van-Zoonen, 2000; Van-Zoonen; Wring, 2012; Meeks, 2012; Fernández-García; Tous-Rovirosa, 2012; Richardson; Parry; Corner, 2013; Campus, 2013; Horn-Sheeler; Vasby-Anderson, 2013).

Los medios de comunicación ejercen una influencia en la representación de la mujer en política, que puede tener consecuencias electorales. La representación de la mujer política en la ficción seriada también contribuye a la conformación del imaginario social, de modo que:

- se trata de una cuestión de máximo interés;

- el aumento de la representación femenina en televisión debería considerarse lógica en relación con la realidad social, lo que se debe analizar es su enfoque y tratamiento;

- es interesante observar si es una representación paralela a la visibilizada en los medios de comunicación o si presenta diferencias significativas.

En la tabla 1 se han clasificado las series políticas occidentales según su ámbito geográfico y modelo político. Con el fin de hacer una preselección de las series a analizar, en la primera fase de esta investigación se observa que: 


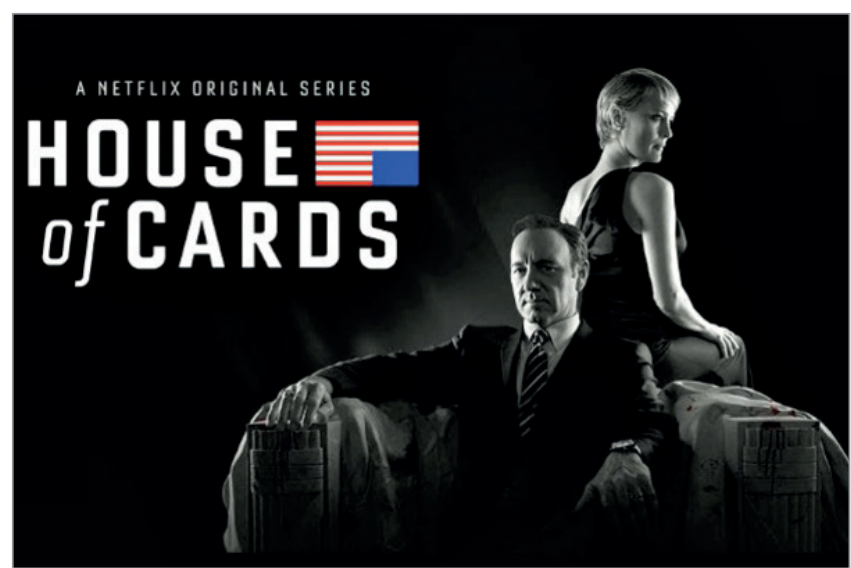

House of cards

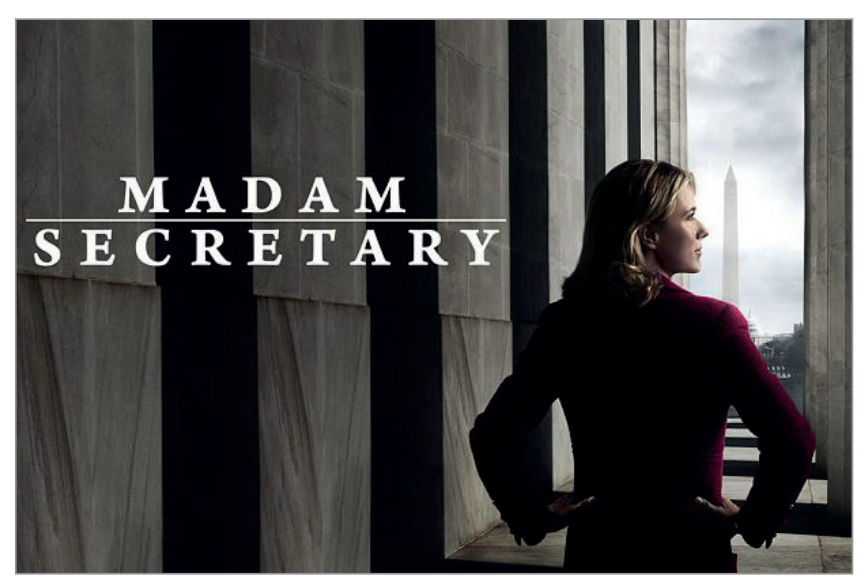

Madam secretary

- desde un punto de vista cuantitativo, las series políticas con protagonismo femenino de mujer política son minoritarias en los tres sistemas, aunque tienen mayor presencia en el liberal y en el anglosajón;

- desde un punto de vista cuantitativo, las series políticas con presencia femenina en calidad de esposa/familiar, son mayoritarias en los tres sistemas;

- el tratamiento de la mujer en las series políticas se caracteriza por: una progresiva mejoría en el enfoque, cada vez más positivo en los sistemas liberales (The good fight, The good wife, Madam secretary) y democrático-corporativos (Ved kongens bord, Borgen), pese a que la focalización de la trama persiste en la compaginación de la vida laboral-familiar y no en la profesional/política en las series protagonizadas por mujeres como actor político (Señora presidenta).
Tabla 2. Ficha de análisis

\begin{tabular}{|l|}
\hline Serie \\
\hline Procedencia de la producción \\
\hline Sistema mediático \\
a. Pluralista polarizado (o del Mediterráneo); \\
b. Liberal (o del Atlántico Norte); \\
c. Democrático-corporativo (o del Centro o del Norte de Europa) \\
\hline Rol temático-narrativo \\
a. Mujeres políticas \\
b. Esposas o familiares de políticos \\
c. Mujeres empresarias (o profesiones liberales) \\
d. Esposas o familiares de empresarios \\
\hline Atribución al cargo y al género (cuestiones de representación mediá- \\
tica y social atribuidas) \\
a. Atribución al cargo, acción centrada en la vida profesional/política \\
b. Atribución al cargo, acción centrada en la vida personal y familiar \\
c. Atribución al cargo, acción centrada en la compaginación entre \\
vida profesional/política y vida personal y familiar \\
\hline Caracterización \\
a. Positiva \\
b. Negativa \\
c. Neutra
\end{tabular}

pertinente investigar si aún persiste en la ficción televisiva a un nivel axiológico una construcción de valores que adscribe los personajes femeninos a una esfera más íntima que pública.

Como hipótesis secundaria, si se produce una representación diferenciada de dichos roles en función de la adscripción geográfica de las series, se observará una presencia cualitativamente más relevante de los personajes y protagonistas femeninas en las series políticas del Centro y Norte de Europa y de acuerdo con el sistema mediático democrático-corporativo (Hallin; Mancini, 2004; 2012).

\section{Los avances de la presencia de la mujer en la política no tienen su correlato en su cobertura mediática}

El análisis temático (Braun; Clarke, 2006) permite identificar las siguientes características socio-semióticas sobre el tipo de presencia de los personajes femeninos (tabla 2). El método consiste en el tratamiento de la información en investigación cualitativa, que permite identificar, organizar, analizar en detalle y reportar patrones o temas a partir de

\section{Metodología}

El presente artículo muestra los resultados de un estudio comparativo entre seis series de subgénero político de producción internacional emitidas en la última década, desde el año 2010 hasta la actualidad.

La hipótesis de trabajo es la siguiente: pese a que la ficción seriada de subgénero político producida a nivel internacional pretende dar mayor presencia y protagonismo a los personajes femeninos para mostrar una voluntad renovadora y de acuerdo con la realidad, es
Tabla 3. Episodios analizados

\begin{tabular}{|c|c|c|c|}
\hline Serie & País, año & Modelo & Episodios \\
\hline Crematorio & España, 2011 & Pluralista polarizado & 8 \\
\hline 1992 & Italia, 2015 & Pluralista polarizado & 10 \\
\hline House of cards & EUA, 2013- & Liberal & 13 \\
\hline Madam secretary & EUA, 2014- & Liberal & 22 \\
\hline Borgen & Dinamarca, 2010-2013 & Democrático corporativo & 10 \\
\hline Bedrag & Dinamarca, 2016- & Democrático corporativo & 10 \\
\hline
\end{tabular}


Tabla 4. Resultados

\begin{tabular}{|c|c|c|c|c|c|c|}
\hline Series & $\begin{array}{l}\text { Procedencia } \\
\text { producción }\end{array}$ & $\begin{array}{l}\text { Sistema } \\
\text { mediático }\end{array}$ & Personaje & $\begin{array}{c}\text { Rol } \\
\text { temático-narrativo }\end{array}$ & $\begin{array}{c}\text { Atribución al cargo y al } \\
\text { género }\end{array}$ & Caracterización \\
\hline 1992 & $\begin{array}{l}\text { Mediterráneo } \\
\text { Italia }\end{array}$ & $\begin{array}{l}\text { Pluralista } \\
\text { polarizado }\end{array}$ & $\begin{array}{l}\text { Verónica } \\
\text { Castello } \\
\text { Bellina }\end{array}$ & $\begin{array}{l}\text { b. Esposa o familiar de } \\
\text { político: } \\
\text { amante de un diputa- } \\
\text { do de la Liga Norte }\end{array}$ & $\begin{array}{l}\text { c. Acción centrada en la } \\
\text { compaginación entre } \\
\text { vida profesional/política } \\
\text { y vida personal y familiar } \\
\text { (inversión)* }\end{array}$ & $\begin{array}{l}\text { Negativa: ambición, cosifi- } \\
\text { cación, mujer objeto }\end{array}$ \\
\hline 1992 & $\begin{array}{l}\text { Mediterráneo } \\
\text { Italia }\end{array}$ & $\begin{array}{l}\text { Pluralista } \\
\text { Polarizado }\end{array}$ & $\begin{array}{l}\text { Bibi Mai- } \\
\text { naghi }\end{array}$ & $\begin{array}{l}\text { b. Esposa o familiar de } \\
\text { político: } \\
\text { hija del político } \\
\text { corrupto Michele } \\
\text { Mainaghi }\end{array}$ & $\begin{array}{l}\text { c. Acción centrada en la } \\
\text { compaginación entre vida } \\
\text { profesional/política y vida } \\
\text { personal y familiar }\end{array}$ & $\begin{array}{l}\text { Negativa: evolución del } \\
\text { personaje: de falta de } \\
\text { interés por los negocios } \\
\text { familiares a adoptar los } \\
\text { métodos de su padre (am- } \\
\text { bición, falta de escrúpulos) }\end{array}$ \\
\hline Crematorio & $\begin{array}{l}\text { Mediterráneo } \\
\text { España }\end{array}$ & $\begin{array}{l}\text { pluralista } \\
\text { polarizado }\end{array}$ & $\begin{array}{l}\text { Sílvia } \\
\text { Bertomeu }\end{array}$ & $\begin{array}{l}\text { b. Esposa o familiar de } \\
\text { político: hija de Rubén } \\
\text { Bertomeu } \\
\text { c. Mujer profesional } \\
\text { liberal (galerista de } \\
\text { arte). }\end{array}$ & $\begin{array}{l}\text { c. Acción centrada en la } \\
\text { compaginación entre vida } \\
\text { profesional/política y vida } \\
\text { personal y familiar }\end{array}$ & $\begin{array}{l}\text { Neutra: evolución del } \\
\text { personaje: del desinterés } \\
\text { por los negocios de su } \\
\text { padre pasa a beneficiarse } \\
\text { de ellos }\end{array}$ \\
\hline Crematorio & $\begin{array}{l}\text { Mediterráneo } \\
\text { España }\end{array}$ & $\begin{array}{l}\text { Pluralista } \\
\text { polarizado }\end{array}$ & Mónica & $\begin{array}{l}\text { b. Esposa o familiar de } \\
\text { político: joven amante } \\
\text { de Rubén Bertomeu } \\
\text { y futura madre de un } \\
\text { nuevo heredero }\end{array}$ & $\begin{array}{l}\text { b. Acción centrada en la } \\
\text { vida personal y familiar }\end{array}$ & $\begin{array}{l}\text { Negativa: ambición, cosifi- } \\
\text { cación, mujer objeto }\end{array}$ \\
\hline $\begin{array}{l}\text { House of } \\
\text { cards }\end{array}$ & $\begin{array}{l}\text { Atlántico Norte } \\
\text { EUA }\end{array}$ & Liberal & $\begin{array}{l}\text { Claire Un- } \\
\text { derwood }\end{array}$ & $\begin{array}{l}\text { b. Esposa o familiar } \\
\text { de político: primera } \\
\text { dama, esposa de } \\
\text { Frank Underwood }\end{array}$ & $\begin{array}{l}\text { c. Acción centrada en la } \\
\text { compaginación entre vida } \\
\text { profesional/política y vida } \\
\text { personal y familiar } \\
\text {-Dirige una organización } \\
\text { sin ánimo de lucro }\end{array}$ & $\begin{array}{l}\text { Negativa (ambición des- } \\
\text { mesurada), } \\
\text {-Trasunto de Hillary Clin- } \\
\text { ton (maldad) }\end{array}$ \\
\hline $\begin{array}{l}\text { Madam } \\
\text { secretary }\end{array}$ & $\begin{array}{l}\text { Atlántico Norte } \\
\text { EUA }\end{array}$ & Liberal & $\begin{array}{l}\text { Elizabeth } \\
\text { McCord }\end{array}$ & $\begin{array}{l}\text { a. Mujer política: } \\
\text { secretaria de Estado }\end{array}$ & $\begin{array}{l}\text { c. Acción centrada en la } \\
\text { compaginación entre vida } \\
\text { profesional/política y vida } \\
\text { personal y familiar }\end{array}$ & $\begin{array}{l}\text { Positiva. profesionalidad } \\
\text { sin renunciar al ámbito } \\
\text { personal } \\
\text {-Trasunto de Hillary Clin- } \\
\text { ton (inteligencia, decisión) }\end{array}$ \\
\hline Borgen & $\begin{array}{l}\text { Centro o Norte } \\
\text { de Europa } \\
\text { Dinamarca }\end{array}$ & $\begin{array}{l}\text { Democrático- } \\
\text { corporativo }\end{array}$ & $\begin{array}{l}\text { Brigitte } \\
\text { Nyborg }\end{array}$ & $\begin{array}{l}\text { a. Mujer política: } \\
\text { primera ministra }\end{array}$ & $\begin{array}{l}\text { c. Acción centrada en la } \\
\text { compaginación entre vida } \\
\text { profesional/política y vida } \\
\text { personal y familiar }\end{array}$ & $\begin{array}{l}\text { Positiva. Profesionalidad } \\
\text { sin renunciar al ámbito } \\
\text { personal } \\
\text {-De la utopía a la real po- } \\
\text { litik. Comete errores, pero } \\
\text { es inteligente y decidida }\end{array}$ \\
\hline Bedrag & $\begin{array}{l}\text { Centro o Norte } \\
\text { de Europa } \\
\text { Dinamarca }\end{array}$ & $\begin{array}{l}\text { Democrático- } \\
\text { corporativo }\end{array}$ & $\begin{array}{l}\text { Claudia } \\
\text { Moreno }\end{array}$ & $\begin{array}{l}\text { c. Mujer profesional } \\
\text { liberal: joven abogada }\end{array}$ & $\begin{array}{l}\text { a. Acción centrada en la } \\
\text { vida profesional/política }\end{array}$ & $\begin{array}{l}\text { Negativa (inteligente pero } \\
\text { demasiado ambiciosa). } \\
\text { Separada, con un hijo. La } \\
\text { cercanía con su jefe le per- } \\
\text { judica (profesionalmente } \\
\text { hablando) }\end{array}$ \\
\hline
\end{tabular}

*Por inversión nos referimos a la mezcla de la vida personal y profesional con el objetivo de ascender socialmente.

una cuidadosa lectura de la información recogida, para inferir resultados que propicien la adecuada comprensión/ interpretación del fenómeno en estudio. La posición que se adopta para analizar los productos televisivos consiste en complementar las mencionadas premisas de Hallin y Mancini $(2004 ; 2012)$ por lo que se refiere al ámbito geopolítico de las producciones con el análisis socio-semiótico (rol temático-narrativo y atribución al cargo y al género). En relación con la caracterización (positiva, negativa, neutra), se establecen tres opciones que se justifican en el análisis de las series.

\subsection{Corpus de análisis}

Con el criterio de acotar producciones de la década actual, se ha escogido la primera temporada de cada serie para mantener un equilibrio en la muestra y dos ejemplos de cada sistema mediático, de acuerdo con Hallin y Mancini (2004; 2012). Así, se analizan series representativas de los modelos mediterráneo polarizado (Crematorio y 1992), democrático corporativo (Borgen y Bedrag) y liberal (House of cards y Madam secretary). La muestra de análisis es resultado de la preselección (véase apartado 3). Se han elegido producciones de la presente década (todas han iniciado sus 


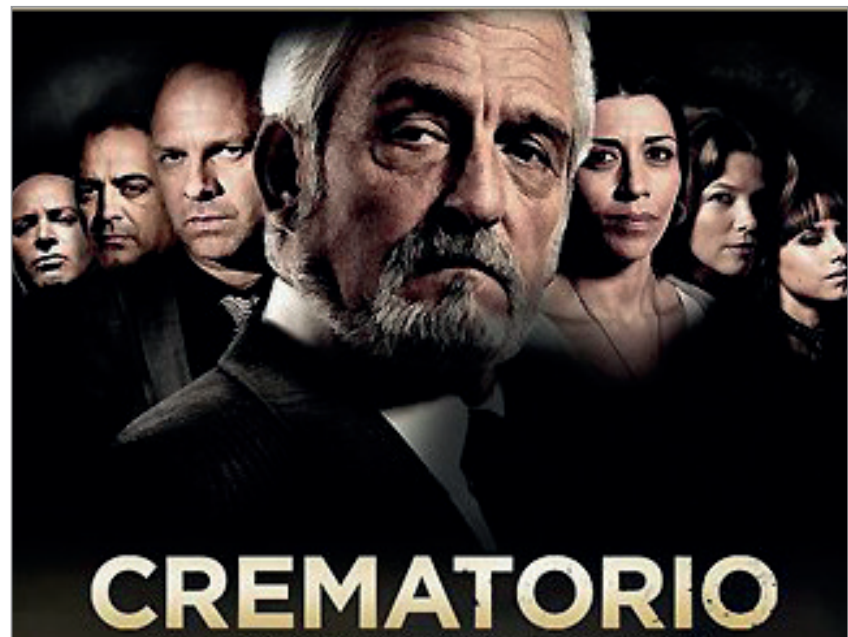

UNA SERIE SOBRE LA CORRUPCIÓN EN ESPAÑA

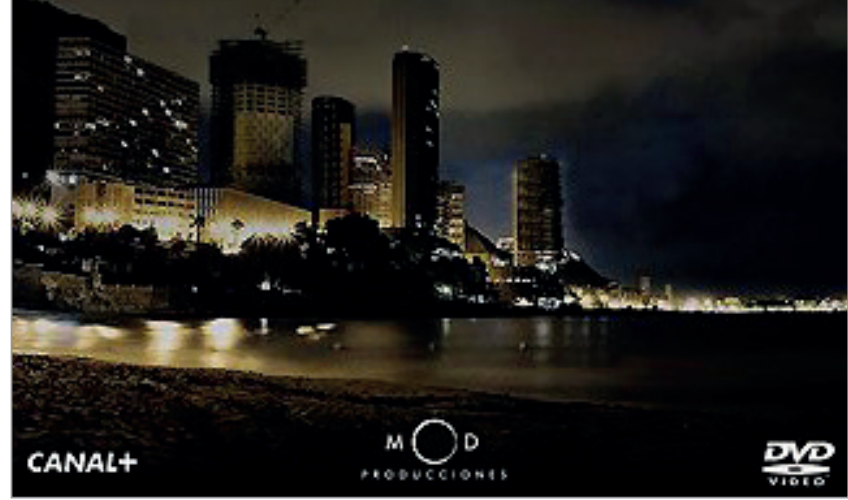

Crematorio

emisiones entre 2010 y 2016), pertenecen al subgénero político, son representativas de sus respectivos países y modelos geopolíticos, y en ellas la mujer está suficientemente representada para posibilitar el análisis. La tabla 3 muestra la procedencia de los 73 episodios analizados, de una hora de duración cada uno.

Una de las principales diferencias en la caracterización de los personajes femeninos se establece entre los sujetos activos de los modelos liberal y democrático corporativo (Borgen, Bedrag, Madam Secretary) y los sujetos pasivos del modelo mediterráneo (1992, Crematorio)

\section{Resultados}

\subsection{Presencia y tipos de personajes femeninos en re- lación con los núcleos de poder}

Los resultados indican que en las series analizadas hay una escasa presencia ${ }^{2}$ de mujeres políticas protagonistas, que corresponden al sistema mediático democrático corporativo (Dinamarca, Borgen protagonizado por la primera ministra) y al sistema anglosajón (EUA, Madam secretary, protagonizado por la secretaria de estado). En el caso de House of cards entendemos que la co-protagonista Claire Underwood

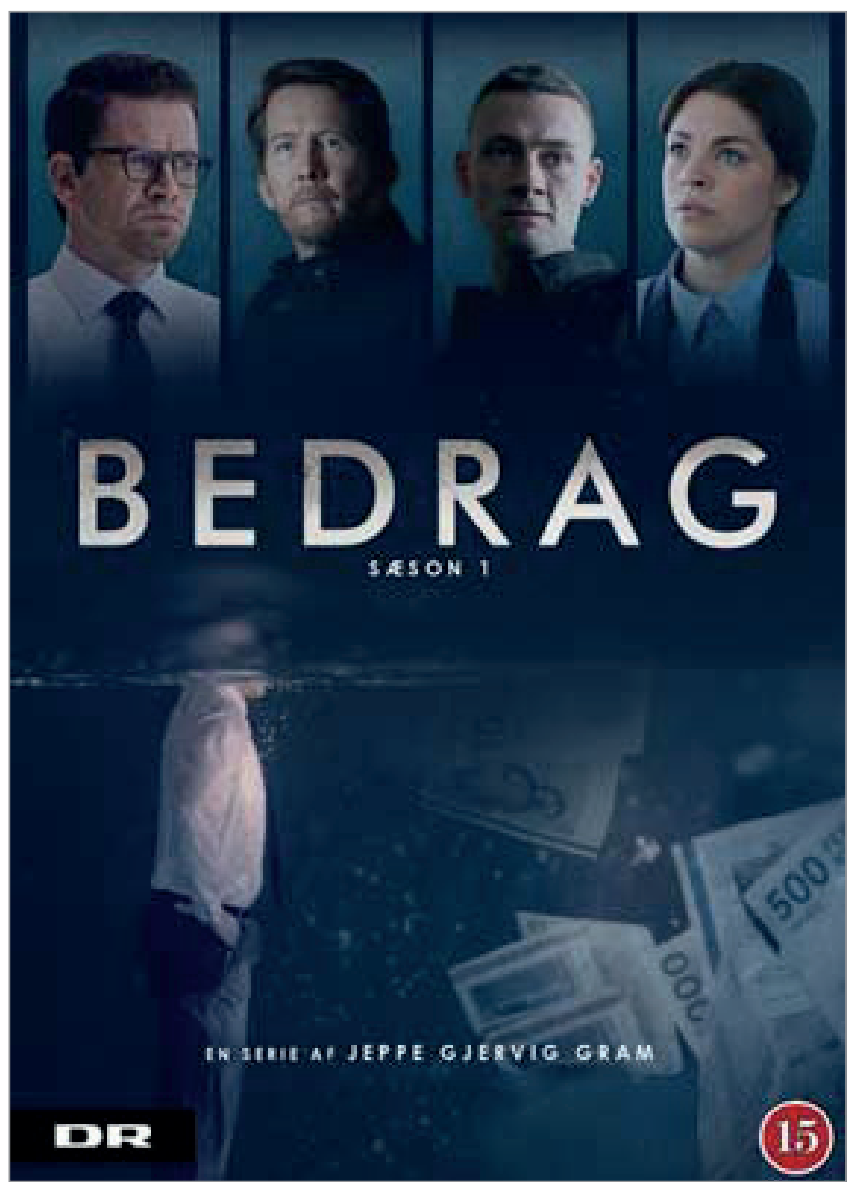

Bedrag

(Robin Wright) ocupa más un rol como esposa del político (en la primera temporada no ejerce cargo político).

La caracterización de los personajes femeninos tiende a ser más negativa que positiva, una atribución que habitualmente se incrementa a medida que la esfera de poder del personaje aumenta (Lacalle; Gómez, 2016).

Es el caso de Crematorio, retrato de una estructura social patriarcal que acaba precisamente con el relevo posible de las corruptelas a manos de la hija del patriarca, algo que reencontramos en 1992. Las series del modelo mediterráneo comparten la estructura mencionada, que va a ser heredada por la hija del protagonista. Aunque con caracterizaciones distintas de Bibi Mainaghi (negativa) y Silvia Bertomeu (neutra). Mediante estas tramas y el embarazo de Mónica en Crematorio se constata que en el sistema mediático mediterráneo la corrupción se hereda. De hecho, una de las características del modelo según Hallin y Mancini (2004) es el clientelismo, por lo que sería interesante ahondar en dicha relación de conceptos.

La ambición marca negativamente a las mujeres. Es la principal característica negativa con la que se retrata a las mujeres profesionales en general (Claudia Moreno), y políticas en particular, así como a esposas (Claire Underwood), amantes (Monica, Veronica Castello) e hijas (Bibi Mainaghi). $Y$ aunque no la hayan ejercido directamente, su proximidad se traduce en un resultado punitivo, con el castigo de la soledad. 
En el último episodio de Crematorio todos los personajes femeninos deben vivir en absoluta soledad un nuevo destino, marcado por la desaparición (marcadamente literal -muerte, separación matrimonial, encarcelamiento-) de los personajes masculinos que les daban un anclaje en la trama de la serie, y por tanto el ámbito político y social "real" en el que ellas solas no existirían.

La narrativa de 1992 apunta la posibilidad para las dos protagonistas femeninas de redimirse gracias al amor, dentro de una dramaturgia que explicita la sexualidad arrebatada, según un tópico muy latino. Pero el modelo trágico se reafirma y la supuesta redención se desvanece. Podría parecer que ambas dominan su destino y deciden sobrevivir en solitario. Pero los atributos de su caracterización no son positivos, a pesar de que el retrato que se hace de la bellina Verónica Castello se apoya a menudo en su mirada herida por los abusos y la prostitución y resulta emocionalmente más proyectivo para el público que la frialdad con la que se dibuja a Bibi Mainaghi como heredera del sistema corrupto. El sistema mediático mediterráneo está claramente representado en varias tramas que siempre confluyen en la corrupción.

Las protagonistas en las series políticas del Centro y Norte de Europa (sistema mediático democrático-corporativo) muestran una presencia cualitativamente más relevante

Tampoco la ambición política o profesional resulta en absoluto simpática en los personajes femeninos de series del Atlántico Norte, como es el caso de Claire Underwood en el retrato shakesperiano que es House of cards. Se ha identificado en numerosas ocasiones a Claire Underwood como una Lady Macbeth de la Casa Blanca. Y también con Hillary Clinton, algo en lo que coincide con la Secretaria de Estado Elizabeth McCord (Téa Leoni) de Madam Secretary. Pero si bien en este último caso el paralelismo se centra en atributos positivizadores como la inteligencia en el ejercicio del poder, en el caso de Claire Underwood como trasunto de $\mathrm{Hi}-$
Ilary Clinton, se asocia a una ambición desmesurada, incluso a la maldad (Waterson, 2015).

\section{Borgen y Madam secretary son los casos} más representativos de un liderazgo de mujeres inteligentes y atractivas, que no se representan con el estereotipo de la feminidad sexy

Sin embargo, esa misma ambición y malas artes se han señalado como el guiño de la audiencia a la audacia del protagonista masculino en su aspiración política, por su actitud ganadora y habilidad para conseguir lo que se proponga (Keller, 2015, p. 120).

La excepción son las series del Norte de Europa, donde esa ambición no "penaliza" al personaje sino que se entiende como el necesario compromiso por el bien común (a pesar de la separación matrimonial de la protagonista, como en Borgen). Borgen y Madam secretary, ésta perteneciente al modelo liberal, son los casos más representativos de un liderazgo de mujeres inteligentes y atractivas, que no se representan con los atributos del estereotipo de la feminidad sexy.

Incluso en Bedrag la ambición profesional exige a la abogada protagonista cambiar sus tejanos por "trajes de poder" (tal y como se describen en la serie), y la transformación física amenaza también con renuncias a la ética personal y a su vida personal y familiar, representada por el hijo que comparte con su expareja.

En resumen, se confirma la hipótesis principal, dado que, a pesar de una mayor presencia y protagonismo de los personajes femeninos, aún persiste en las series políticas a un nivel axiológico una construcción de valores que adscribe los personajes femeninos a una esfera más íntima que pública y que presenta variaciones sustanciales en función del sistema político al que se adscribe cada serie:

- caracterización negativa;

- ambición como contravalor;

- belleza física como atributo de poder;

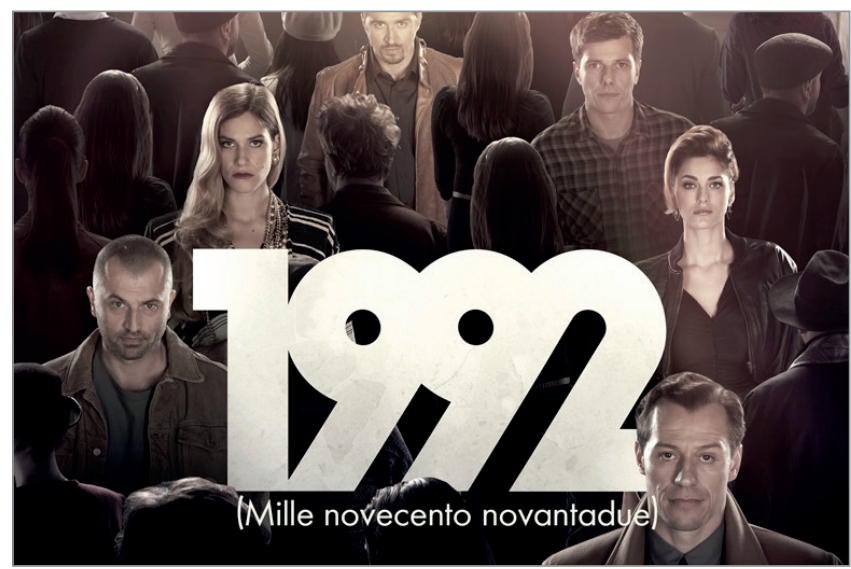

1992

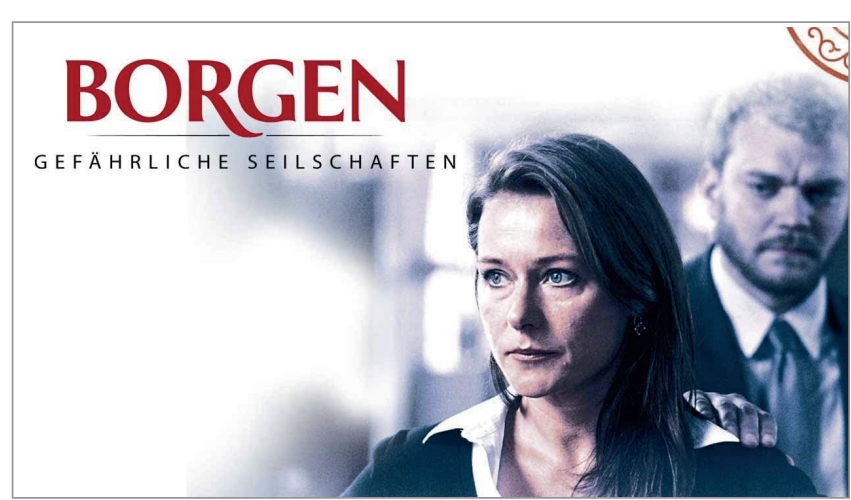

Borgen 
- soledad -presentada como renuncia o abandono de los personajes masculinos- como penalización.

Se confirma la hipótesis secundaria ya que las protagonistas y personajes femeninos en las series políticas del Centro y Norte de Europa -sistema mediático democrático-corporativo (Hallin; Mancini, 2004, 2012)- muestran una presencia cualitativamente más relevante. Como se detalla en el análisis temático, los arquetipos de masculinidad y feminidad están más anclados tanto en los sistemas mediáticos mediterráneos -con una representación trágica que se contextualiza en tramas de un pasado reciente- como en el atlántico, donde incluso la aproximación a una secretaria de estado se mantiene en la dicotomía espacio público/privado. En Madam secretary se destaca la dualidad de la política protagonista al centrar la trama, como en Borgen, y supone una relevante mejora respecto a Señora presidenta. En Bedrag, la contradicción de una "ecología sucia" está encarnada por los dilemas éticos de la abogada Claudia Moreno (Natalie Madueño). Recordemos en el primer episodio su frase post-it de cabecera: "Si tus sueños no te asustan, no son lo bastante grandes".

Sin ninguna duda el caso de Borgen resulta la descripción de una mujer política con grandes ideales que en el ejercicio del poder debe olvidarlos, como se ejemplifica con la política de

Del total de personajes analizados, más del $50 \%$ son caracterizados de modo negativo

pactos, a causa de la real politik. Nos parece que la voluntad de realismo de esta producción emblemática del modelo democrático- corporativo (del Norte de Europa) anticipa -y concentra en una protagonista femenina- lo que la realidad política y social muestra: el difícil equilibrio entre el espacio privado y el espacio público en la era de la hipervisibilidad, acentuado aún más en el caso de las mujeres profesionales que ocupan puestos de responsabilidad. Cabe resaltar que la serie supuso una proyección al mundo occidental de una de las claves del modelo democrático-corporativo, no sólo con el personaje de Brigitte Nyborg (Sidse Babett Knudsen), sino también el de su marido, Philip (Mikael Birkkjær).

En el modelo liberal se parte de una representación de la mujer bastante secundaria en las series políticas protagonizadas por hombres (The thick of it), o de la equiparación de la mujer política con su vida personal (Señora presidenta) hasta llegar a algo próximo a la equidad, a una situación en la que hombres y mujeres se aproximan por igual al poder (Borgen, Madam secretary, The good wife, The good fight) y en la que padecen las consecuencias y la transformación profesional y personal que dicho poder conlleva también de manera ecuánime entre géneros (Borgen).

\section{Conclusiones}

En el caso específico de las series políticas, nos parece de gran importancia la influencia de los imaginarios sociales (Aran-Ramspott; Rodrigo-Alsina, 2012) a la hora de transmitir a las nuevas generaciones un sesgo de género en relación tanto al ejercicio profesional en general como al político en particular. Hemos observado que una mayor concordancia con la realidad social (y en algún caso, con una intención manifiesta contraria a la perpetuación de mecanismos de invisibilidad o prejuicios de género) convive con la persistencia de determinados estereotipos. Efectivamente, los sistemas mediáticos mediterráneos y atlánticos incorporan muy tímidamente en la ficción a políticas protagonistas (Madam secretary, The good fight, la próxima temporada de Homeland), pero siguen depositando en los personajes femeninos la tensión -con elementos de renuncia- de los ámbitos profesionales y personales/familiares. Esta polarización, si bien no parece haberse resuelto en el espacio del Norte de Europa, sí que permite un diseño más contradictoriamente humano de los personajes femeninos, a la vez que muestra modelos de masculinidad más acorde con los tiempos actuales (por ejemplo, el padre policía en Bedrag).

De las seis series, con ocho personajes femeninos analizados, observamos que de un modo mayoritario su representación responde a la conciliación profesional y personal (c), mientras que sólo una se centra en lo personal y familiar (b) y también sólo una se centra únicamente en lo profesional (a).

Del total de personajes, cinco son caracterizados de modo negativo, dos en positivo y uno neutro. Sobre los personajes con caracterización negativa, ésta se produce a causa de la ambición o de la cosificación-mujer objeto, de modo más preeminente en las series analizadas pertenecientes al modelo mediterráneo. Los dos personajes caracterizados como positivos responden a una atribución al cargo de compaginación de la vida familiar y laboral (Madam secretary y Borgen). En definitiva, una de las principales diferencias en la caracterización de los personajes femeninos se establece entre los sujetos activos propios de los modelos liberal y democrático corporativo (Borgen, Bedrag, Madam secretary) y los sujetos pasivos del modelo mediterráneo (1992, Crematorio). Entre las mujeres que toman las riendas y cometen sus propios errores y las que viven a la sombra de los hombres y heredan sus errores.

\section{Notas}

1. Los asesores de las series de ficción surgen en los Estados Unidos a mediados de siglo pasado con la simbiosis entre la American Medical Association y las productoras televisivas. Dicha función se sigue ejerciendo en las series de profesiones, como en El ala oeste con Marlin Fitzwater, Dee Dee Myers o Patrick K. Caddell.

2. Cabe destacar la campaña, en enero de 2016, de políticas catalanas protestando por el tratamiento machista recibido en los medios de comunicación.

https://goo.gl/3Z9WrU

\section{Bibliografía}

Ang, len (1985). Watching Dallas. Soap opera and the melodramatic imagination. Londres: Methuen. ISBN: 9780 415045988

Aran-Ramspott, Sue; Medina-Bravo, Pilar; Rodrigo-Alsina, Miquel (2011). "Management of emotions in American fiction series: When being (and feeling) woman sells". International journal of arts and technology, v. 4, n. 1, pp. 6-18. https://doi.org/10.1504/IJART.2011.037766 
Aran-Ramspott, Sue; Medina-Bravo, Pilar; Rodrigo-Alsina, Miquel; Munté-Ramos, Rosa-Auria (2014). "New fictional constructions of masculinity in love relationships: A case study of the Catalan TV series Porca Miseria". Catalan journal of communication and cultural studies, v. 6, n. 1, pp. 75-94. https://doi.org/10.1386/cjcs.6.1.75_1

Aran-Ramspott, Sue; Rodrigo-Alsina, Miquel (2012). “Children's interpretation of violence in television programmes: The notion of proximity realism". Culture and education, v. 24, n. 4, pp. 489-504.

https://doi.org/10.1174/113564012803998794

Braun, Virginia; Clarke, Victoria (2006). "Using thematic analysis in psychology". Qualitative research in psychology, v. 3, n. 2, pp. 77-101.

http://eprints.uwe.ac.uk/11735/2/thematic_analysis_revised... https://doi.org/10.1191/1478088706qp063oa

Campus, Donatella (2013). Women political leaders and the media. New York: Palgrave MacMillan. ISBN: 9781 137295545

Davis, Donald M. (1990). "Portrayals of women in prime-time network television: Some demographic characteristics". Sex roles, v. 23, n. 5-6, pp. 325-332.

https://goo.gl/mD8WA3

https://doi.org/10.1007/BF00290052

Durkin, Kevin (1985). Television and sex-role acquisition 1: Content. British journal of social psychology, v. 24, pp. 101-113. https://www.researchgate.net/publication/19267549_ Television and Sex-Role Acquisition 1 Content https://doi.org/10.1111/j.2044-8309.1985.tb00669.x

Engelstad, Audun (2008). "Watching politics. The representation of politics in primetime television drama". Nordicom review, v. 29, n. 2, pp. 309-324.

http://www.nordicom.gu.se/sites/default/files/kapitelpdf/269_engelstad.pdf

Fernández-García, Núria; Tous-Rovirosa, Anna (2012). “La representación de las mujeres políticas en los medios". En: Larrondo-Ureta, Ainara; Meso-Ayerdi, Koldo. $4^{\text {as }}$ Jornadas sobre mujeres y medios de comunicación. Leioa: UPV. ISBN: 9788498605921

https://www.ehu.eus/documents/2007376/2109490/4_ Jornadas_Mujeres_Medios.pdf

Fernández-Villanueva, Concepción; Revilla-Castro, Juan-Carlos; Domínguez-Bilbao, Roberto; Gimeno-Jiménez, Leonor; Almagro, Andrés (2009). "Gender differences in the representation of violence on Spanish television: Should women be more violent?". Sex roles, v. 61, pp. 85-100.

http://eprints.ucm.es/36959

https://doi.org/10.1007/s11199-009-9613-9

Fouts, Gregory; Burggraf, Kimberley (1999). “Television situation comedies: Female body images and verbal reinforcements". Sex roles, v. 40, n. 5-6, pp. 473-481. https://doi.org/10.1023/A:1018875711082

Galán-Fajardo, Elena (2007). La imagen social de la mujer en las series de ficción. Cáceres: Universidad de Extremadura. ISBN: 9788477237211
García-Muñoz, Nuria; Fedele, Madalena; Gómez-Díaz, Xiana (2012). "The occupational roles of television fiction characters in Spain: Distinguishing traits". Comunicación y sociedad, v. 25, n. 1, pp. 349-366

http://dadun.unav.edu/handle/10171/27371

Geraghty, Christine (1991). Women and soap opera: A study of prime time soaps. Polity Press: Cambridge, UK. ISBN: 978 0745604893

Gerbner, George; Gross, Larry; Jackson-Beeck, Marilyn; Jeffries-Fox, Suzanne; Signorielli, Nancy (1978) "Cultural indicators: Violence profile No. 9". Journal of communication, v. 28, n. 3, pp. 176-207.

http://web.asc.upenn.edu/gerbner/Asset.aspx?asset/D=2746 https://doi.org/10.1111/j.1460-2466.1978.tb01646.x

Gordillo-Álvarez, Inma; Guarinos, Virginia (2010). Todos los cuerpos. El cuerpo en televisión como obsesión hipermoderna. Córdoba (Argentina), Babel Ed. ISBN: 9789871520381

Hallin, Daniel C.; Mancini, Paolo (2004). Comparing media systems: Three models of media and politics. Cambridge: Cambridge University Press. ISBN: 0521543088 https://goo.gl/bGg9fH

Hallin, Daniel C.; Mancini, Paolo (2012). Comparing media systems beyond the Western world. Cambridge: Cambridge University Press. ISBN: 9781107699540

Horn-Sheeler, Kristina; Vasby-Anderson, Karrin (2013). Woman president: Confronting postfeminist political culture. College Station, Texas: A\&M University Press. ISBN: 9781 603449830

Keller, James (2015). "The vice in vice president: House of cards and the morality tradition". Journal of popular film and television, v. 43, n. 3, pp. 111-120.

https://goo.gl/wrLx9S

https://doi.org/10.1080/01956051.2015.1027649

Lacalle, Charo; Castro-Mariño, Deborah (2016). “Promotion of Spanish scripted television on the internet: analyzing broadcast-related websites' content and social audience". El profesional de la información, v. 25, n. 2, pp. 246-253.

https://doi.org/10.3145/epi.2016.mar.11

Lacalle, Charo; Gómez, Beatriz (2016). "La representación de las mujeres trabajadoras en la ficción televisiva española". Comunicar, v. 47, n. 24, pp. 59-67.

https://doi.org/10.3916/C47-2016-06

López-García, Guillermo (2015). "Los spin doctors en las arenas de la comunicación política: The thick of it". En: Tous-Rovirosa, Anna. La política en las series de televisión. Entre el cinismo y la utopía. Barcelona: UOC, pp. 173-187. ISBN: 9788490647684

Lull, James (1990). Inside family viewing. Ethnographic research on television's audiences. Londres: Routledge. ISBN: 9780415049979

Medina-Bravo, Pilar; Aran-Ramspott, Sue; Munté-Ramos, Rosa-Auria; Rodrigo-Alsina, Miquel; Guillén-Soler, Montserrat (2010). "La representación de la maternidad en las series de ficción norteamericanas. Propuesta para un análisis de contenido: Desperate housewives y Brothers \& sisters". 
En: Comunicación y desarrollo en la era digital. Congreso AE-IC, 3-4 de febrero de 2010. ISBN: 9788461428182. http://www.aeic2010malaga.org/upload/ok/137.pdf

Meeks, Lindsey (2012). "Is she 'man enough'? Women candidates, executive political offices, and news coverage". Journal of communication, v. 62, pp. 175-193.

https://goo.gl/w1H3A3

https://doi.org/10.1111/j.1460-2466.2011.01621.x

Morley, David (1986). Family television: Cultural power and domestic leisure. Londres: Comedia Publishing Group. ISBN: 0 90689073X

https://monoskop.org/images/9/97/Morley_David_Family Television_Cultural_Power_and_Domestic_Leisure_1988.pdf

Richardson, Kay; Parry, Katy; Corner, John (2013). Political culture and media genre: Beyond the news. New York: Palgrave MacMillan. ISBN: 9781137291271

Sangrador, José-Luis (1996). “Mujer y amor. Una perspectiva psicosocial". En: Blanco, Ana-Isabel (ed.). Mujer, violencia y medios de comunicación. León: Universidad de León, Secretariado de Publicaciones, pp. 117-36. ISBN: 8477195854

Searle, John R. (1975). "Speech acts and recent linguistics". Annals of the New York Academy of Sciences, v. 263, pp. 27-38. https://goo.gl/F58w1P https://doi.org/10.1111/j.1749-6632.1975.tb41567.x

Silverstone, Roger (1994). Televisión y vida cotidiana. Buenos Aires: Amorrortu Editores. ISBN: 9789505186457

Thompson, John (1998). Los media y la modernidad. Una teoría de los medios de comunicación. Barcelona: Paidós. ISBN: 9788449305788
Tous-Rovirosa, Anna (2011). "Los rostros de la mujer en la televisión de los EE.UU. Tradición, feminismo y postfeminismo en las series estadounidenses". En: Larrondo-Ureta, Ainara; Meso-Ayerdi, Koldo. $3^{\text {as }}$ Jornadas sobre mujeres $y$ medios de comunicación. Bilbao: Servicio editorial de la Universidad del País Vasco, pp. 187-202.

Tous-Rovirosa, Anna (2015). La política en las series de televisión. Entre el cinismo y la utopía. Barcelona: UOC. ISBN: 9788490647684

Tous-Rovirosa, Anna (2017). "Series políticas". En: Vilches, Lorenzo. Diccionario de narrativas. Barcelona: Random House Penguin (en prensa).

Tous-Rovirosa, Anna; Meso-Ayerdi, Koldo; Simelio-Solà, Núria (2013). "The representation of women's roles in television series in Spain. Analysis of the Basque and Catalan cases". Comunicación y sociedad, v. 26, n. 3, pp. 67-97. https://www.unav.es/fcom/communication-society/en/ articulo.php?art_id=459

Van-Zoonen, Liesbet (2000). "The personalization of politics: Opportunities for women?. International journal for politics and psychology, v. 9, n. 3-4, pp. 19-35. https://goo.gl/tHQBwh

Van-Zoonen, Liesbet; Wring, Dominic (2012). "Trends in political television fiction in the UK: Themes, characters and narratives, 1965-2009". Media, culture and society, v. 34, n. 3, pp. 263-279. https://goo.gl/6aJK8a

Waterson, Jim (2015). "House of cards creator: Hillary Clinton is the real Claire Underwood". BuzzFeed, July 10. https://goo.gl/SogxXM
La Fundación Biblioteca Social es una institución sin ánimo de lucro que se constituyó en el año 2014. Tiene como objetivo contribuir a compensar los desequilibrios sociales apoyando proyectos que llevan a cabo las bibliotecas públicas, dirigidos a los sectores más vulnerables de la sociedad.

\section{¿Colaboras?}

fundacionbibliotecasocial.org info@fundacionbibliotecasocial.org

3 @Biblio_Social

FundacionBibliotecasSocial

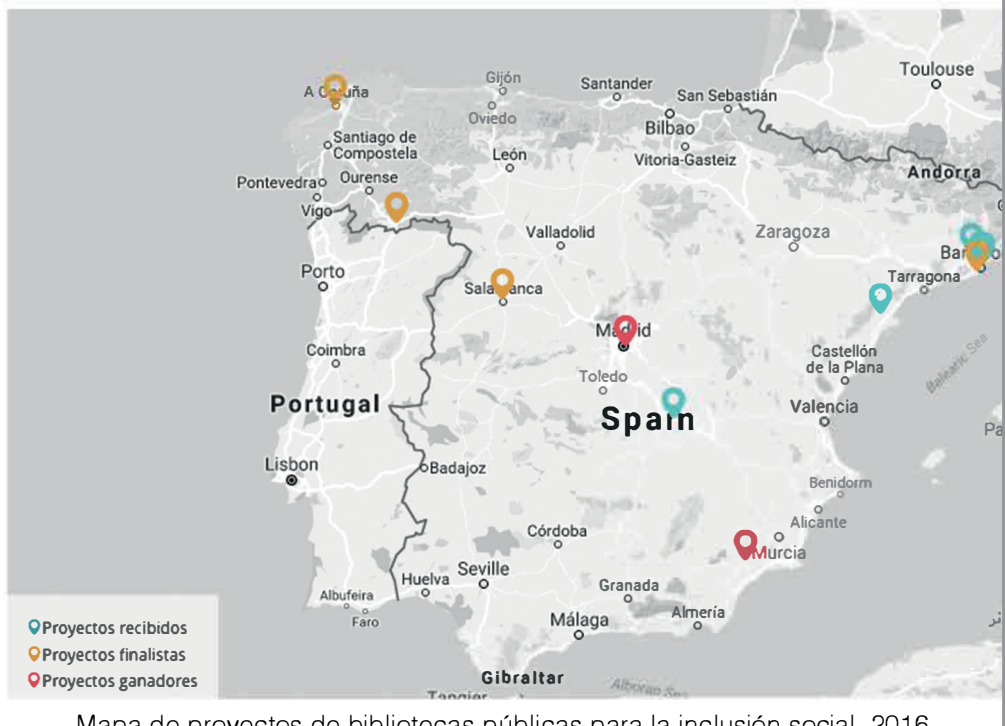

Mapa de proyectos de bibliotecas públicas para la inclusión social. 2016.

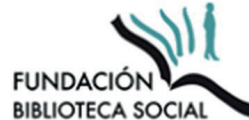

